\title{
Whodas II: la herramienta de la organización mundial de la salud (OMS) para evaluar la discapacidad
}

\author{
Ana Rebollo Rubio, José Antonio González Castillo, Sandra Cabrera Azaña, María José Pinilla Cancelo, \\ Juan José Mancilla Francisco
}

\section{Hospital Regional Carlos Haya. Málaga}

\section{Introducción:}

La Dependencia Funcional (DF), se define como la pérdida de autonomía física, psíquica o intelectual a causa de procesos relacionados con la salud del individuo. Aunque suele asociarse a la edad, la DF no es una situación exclusiva de las personas mayores ya que puede afectar a cualquier sector de la población. Dentro de este contexto el IMSERSO caracteriza la DF como aquella que va a necesitar de la ayuda cuantitativa o cualitativa aportada por un tercero a una persona discapacitada para la realización de sus actividades. Por tanto, la discapacidad es un atributo inseparable de la dependencia, pero no recíproco. La DF es el resultado de una discapacidad, pero no todos los discapacitados tienen DF puesto que no precisan ayuda de terceros. Dentro de nuestra labor como enfermeros de una Unidad de Diálisis Hospitalaria hemos empezado a utilizar el World Health Organization Disability Assessment Schedule version 2 (WHODAS II) que es el instrumento que propone la OMS para la evaluación de la discapacidad.

\section{Objetivo, material y método:}

Estudio epidemiológico de corte transversal para la valoración de discapacidad y dependencia funcional. Comparar los resultados obtenidos mediante el Indice de Barthel (BI) con los obtenidos según el WHODAS II y relacionarlos con una variable predictora de dependencia funcional como el Indice de Charlson (ICC) modificado por edad para evaluar la congruencia de ambas medidas.

\section{Resultados:}

EI BI arrojó una media de 87,5 \pm 19,3 puntos, situándose en la banda del percentil 75, con un rango intercuartiles $63,75-100$ y una mediana de 100. EI WHODAS II obtuvo una media de $31,6 \pm 25,3$, con un rango de 0 (ninguna discapacidad) a 88. El rango intercuartil fue de $12,2-51,3$, con una mediana de 26,6 . Analizando conjuntamente los valores de BI y WHODAS II con el ICC modificado por edad obtuvimos que si bien ambas correlacionan significativamente con la comorbilidad $(p<0,05)$, parece que la DF lo hace con mayor fuerza que la discapacidad.

\section{Conclusiones:}

La medida de la discapacidad y su adscripción y tabulación en una clasificación como la CIF nos permite identificar de forma precoz áreas de discapacidad física, psíquica o relacional que en un futuro pudieran abocarse a DF.

\section{Referencias Bibliográficas}

1. Consejo de Europa. Recomendación R (87) 22 sobre la protección y supervisión de las personas mayores. Disponible en: http://www.hrea.net/learn/ guides/ ancianos.html.

2. BOE núm. 299 LEY 39/2006, de 14 de diciembre, de promoción de la Autonomía Personal y Atención a las personas en situación de Dependencia. Madrid. 2006. 
3. Quejereta González M. Discapacidad/dependencia. Unificación de criterios. Madrid: Ministerio de Trabajo y Asuntos Sociales. IMSERS0, 2003.

4. Boldt C., Grill E., Bartholomeyczik S., Brach M., Rauch A., Eriks-hoogland I., Stucki G. (2010) Combined application of the International Classification of Functioning, Disability and Health and the NANDA International Taxonomy II. Journal of Advanced Nursing 66(8), 1885-1898.

5. Impact of incident comorbidity on functional loss in elderly chronic kidney disease patients undergoing hemodialysis. Mansilla et al. CANNT Journal. January-March 2012, Volume 22, Issue 1. 